\title{
LHC BEAM DUMPING SYSTEM: EXTRACTION CHANNEL LAYOUT AND ACCEPTANCE
}

\author{
B.Goddard, M.Gyr, J.Uythoven, R.Veness, W.Weterings, CERN, Geneva, Switzerland
}

\section{Abstract}

The LHC beam dumping system must safely abort the LHC beams under all conditions, including those resulting from abnormal behaviour of machine elements or subsystems of the beam dumping system itself. The extraction channels must provide sufficient aperture both for the circulating and extracted beams, over the whole energy range and under various beam parameters. These requirements impose tight constraints on the tolerances of various extraction channel components, and also on the allowed range of beam positions in the region of these components. Operation of the beam dumping system under various fault states has been considered, and the resulting apertures calculated. After describing briefly the beam dumping system and the extraction channel geometry, the various assumptions made in the analysis are presented, before deriving tolerance limits for the relevant equipment and beam parameters.

\section{INTRODUCTION}

The beam dumping system is a vital element of the LHC, where $340 \mathrm{MJ}$ of energy is contained in each circulating beam and where a single nominal intensity high energy bunch could melt or damage a metallic surface [1]. The concept of the beam dumping system is to fast-extract the beam in a loss-free way from each ring of the collider and to transport it to an external dump, positioned sufficiently far away to allow for appropriate beam dilution. This requires a particle-free gap in the circulating beam for the rise time of the extraction kicker.

The layout of the system under construction in straight section 6 of the LHC is shown in Figure 1. It comprises for each ring 15 extraction kicker magnets MKD (3 $\mu \mathrm{s}$ rise time, $0.27 \mathrm{mrad}$ horizontal deflection), 15 steel septum magnets MSD (2.4 mrad vertical deflection) and 10 modules of dilution kicker magnets MKB. The beam dump proper, situated in a cavern $750 \mathrm{~m}$ from the centre of the septum magnets, comprises the TDE core and shielding (total weight about 1000 tons).

\section{BEAM PARAMETERS}

The beam dumping system must be able to accept LHC beams with nominal parameters up to ultimate intensity (e.g. during a planned abort at the end of a physics run) and also beams with off-normal parameters (e.g. as arising from an equipment failure or beam instability), in addition to variations imposed by optical effects (e.g. beta-beating, tuning range). The beam characteristics are well known for the LHC nominal beam; however, the characteristics of the planned commissioning and early years beams are also taken into account, as for completeness is the ultimate beam. The relevant worstcase beam characteristics (LHC V6.4) to be expected at point 6 have been presented [2] and are given in table 1 .

Table 1. Maximum assumed LHC beam characteristics.

\begin{tabular}{|c|c|c|c|c|c|}
\hline \multirow[t]{2}{*}{ Beam } & \multicolumn{2}{|c|}{$\operatorname{Max} \varepsilon_{n}$} & \multirow{2}{*}{$\begin{array}{c}\text { Total } \\
\text { Orbit } \\
\text { mm }\end{array}$} & \multirow{2}{*}{$\begin{array}{c}\text { Beta } \\
\text { variation } \\
\%\end{array}$} & \multirow{2}{*}{$\begin{array}{c}\text { Tota } \\
\text { p+ } \\
10^{14}\end{array}$} \\
\hline & $\begin{array}{c}450 \mathrm{GeV} \\
\mu \mathrm{m}\end{array}$ & $\begin{array}{c}7 \mathrm{TeV} \\
\mu \mathrm{m}\end{array}$ & & & \\
\hline Commission & 6.0 & 12.0 & \pm 4 & 42 & 0.3 \\
\hline Early Years & 6.0 & 12.0 & \pm 4 & 42 & 0.8 \\
\hline Nominal & 7.5 & 15.0 & \pm 4 & 42 & 3.1 \\
\hline Ultimate & 7.5 & 15.0 & \pm 4 & 42 & 5.3 \\
\hline
\end{tabular}

\section{FAILURE MODES}

The beam dumping system acts at the request of the machine protection system, which collects the status and messages from all critical machine subsystems. Although great effort is undertaken in maximising the reliability of the dumping system (e.g., appropriate margins, redundancy, autonomy, failure tolerant signal transmission, and by monitoring of all vital parameters), failures cannot be totally excluded [3].

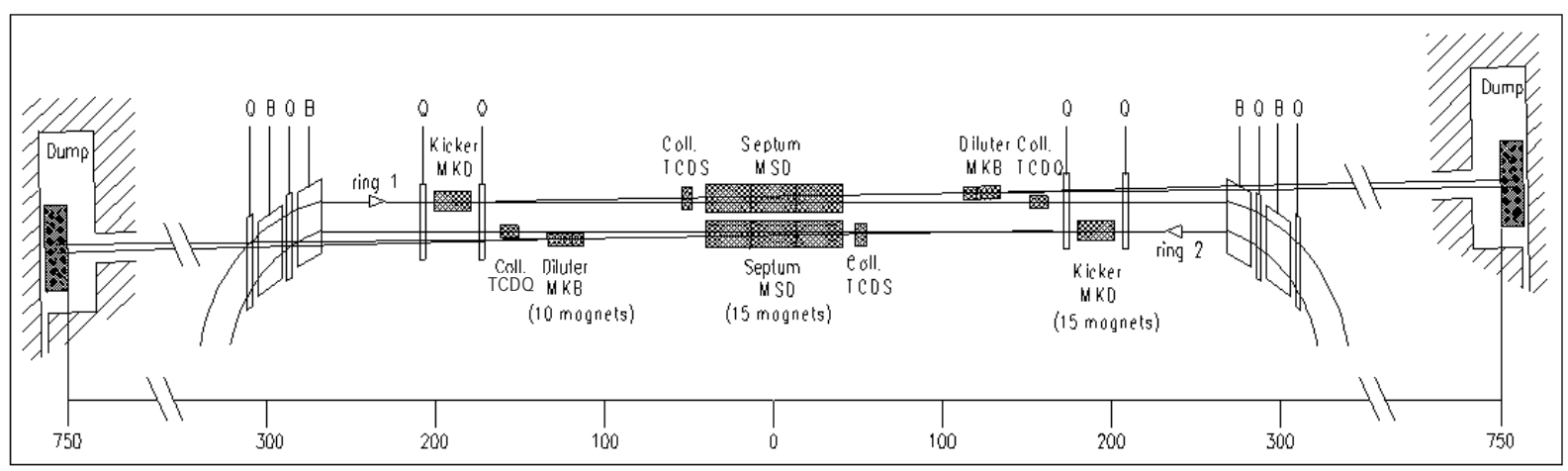

Figure 1. Schematic layout of beam dumping system elements. 
One serious failure is an unsynchronised firing of the extraction kickers [4], which would damage the steel septum and vacuum chamber. Protective elements (TCDS and TCDQ), are therefore placed in front of the septum and the Q4 quadrupole downstream of the septum [5].

The other relevant internal beam dumping system failure mode is the 'missing module' case, where only 14 out of the 15 MKD modules trigger.

The effects of off-normal operating conditions arising from the LHC machine proper are grouped together and treated under local orbit excursions and emittance growth.

\section{APERTURE FOR CIRCULATING BEAM}

From [6], for the normal machine the specification to be met is $n_{1} \geq 7.0$. For this analysis an extra factor 1.17 is added to the $\beta$ for variations in the LHC tune.

\section{At TCDS}

The TCDS is positioned as far in as possible to the circulating beam axis. Assuming mechanical and alignment tolerances $\delta_{\mathrm{xT}}= \pm 1 \mathrm{~mm}$ for the TCDS, with a maximum orbit of $+2 \mathrm{~mm}$, the curve of available aperture versus orbit is shown in Figure 2.

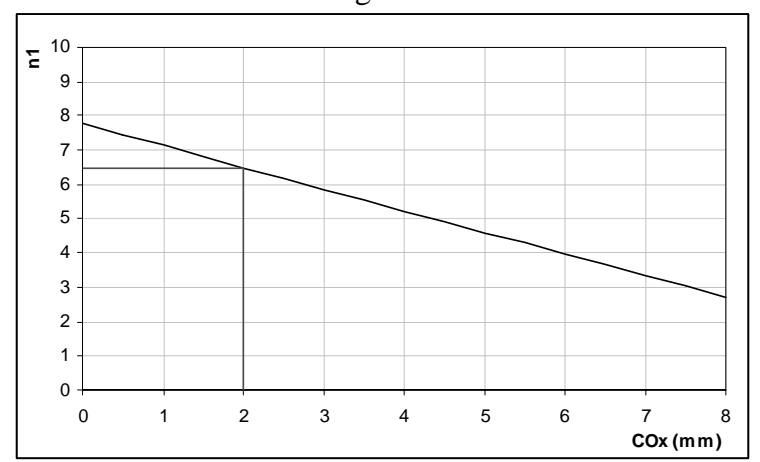

Figure 2. Aperture at TCDS for circulating beam as a function of local beam position.

\section{At MSDC vacuum chamber}

The outside position of the chamber is $+28.2 \mathrm{~mm}(-20.2$ inside), which provides a fairly comfortable aperture for the circulating beam, where at an orbit excursion of $+4 \mathrm{~mm}$ the aperture remains above $\mathrm{n} 1=6.5$.

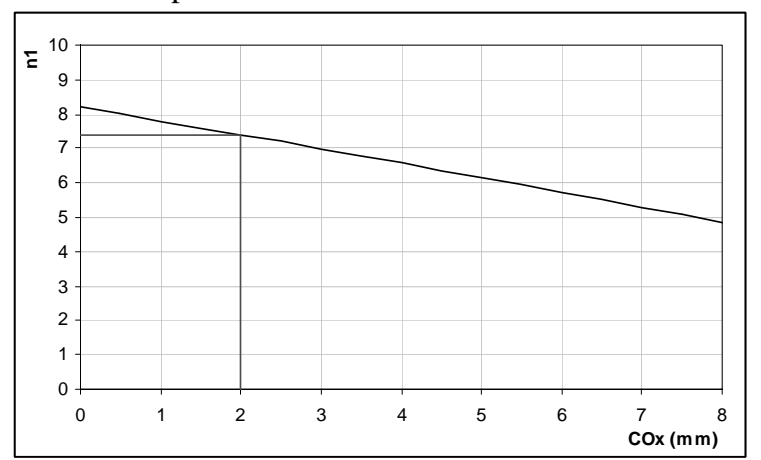

Figure 3. Circulating beam aperture at MSDC5 vacuum chamber as a function of local horizontal beam position.

\section{APERTURE FOR EXTRACTED BEAM UNDER NORMAL CONDITIONS}

The width of the MSD septum and the assumed closed orbit errors define the geometrical shape of the TCDS protection element. The total width of the MSDC septum and vacuum chambers (including tolerances) is $28.3 \mathrm{~mm}$. The static closed orbit is assumed to be held to $\pm 2 \mathrm{~mm}$. For the aperture calculation the total overshoot of the MKD kicker waveform is taken to be $10 \%$ [7].

\section{Nominal case - 15/15 MKD}

The extracted beam is centred in the septum gap for the nominal case. The same kick is imposed from $450 \mathrm{GeV}$ to $7 \mathrm{TeV}$ beam energy. The available apertures as a function of orbit are shown in figures 4 and 5. Assuming that for 'loss free' extraction an aperture of $4 \sigma$ is sufficient at $450 \mathrm{GeV}$, and $6 \sigma$ at $7 \mathrm{TeV}$, the respective maximum orbit excursion should stay within about \pm 4.2 and $\pm 7.5 \mathrm{~mm}$.

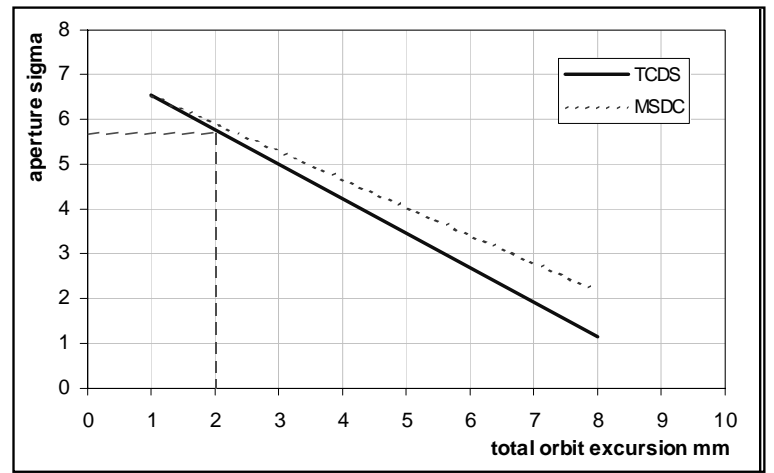

Figure 4. Aperture at $450 \mathrm{GeV}$ for extracted beam as a function of the orbit in the nominal $15 / 15$ MKD case.

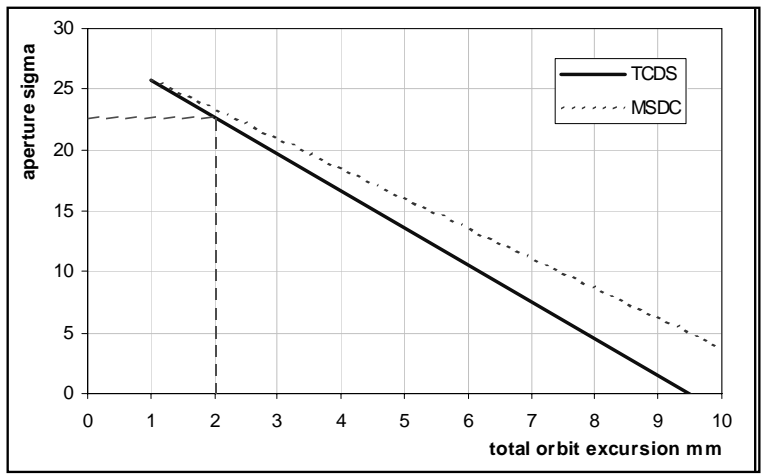

Figure 5. Aperture at $7 \mathrm{TeV}$ for extracted beam as a function of the orbit in the nominal 15/15 MKD case.

\section{Missing module case - 14/15 MKD}

The worst missing MKD module is the MKD1; in this case the deflection at the TCDS is $91.96 \%$ of the total, and the beam approaches the TCDS. The available apertures at $450 \mathrm{GeV}$ and $7 \mathrm{TeV}$ as a function of orbit are shown in figures 6 and 7. Here, for even moderate orbit excursions, the TCDS will receive beam in the event of an MKD missing, with the attendant risk of quenches in downstream super-conducting magnets or TCDS damage. 


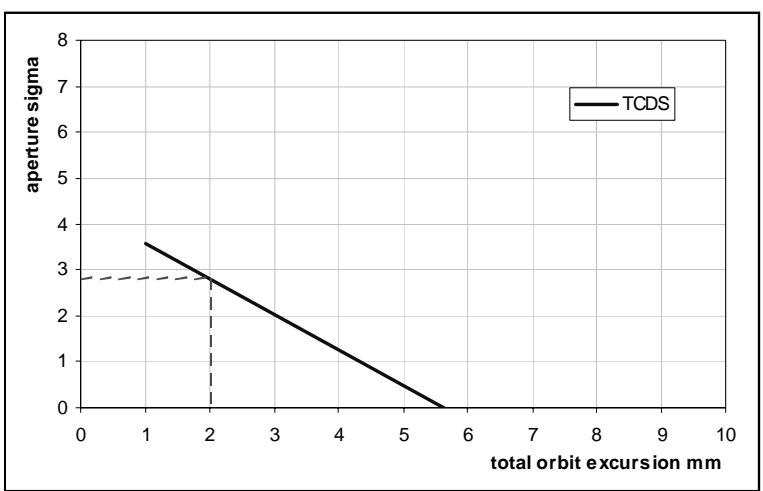

Figure 6. Aperture at $450 \mathrm{GeV}$ for extracted beam as a function of the orbit in the 14/15 MKD case.

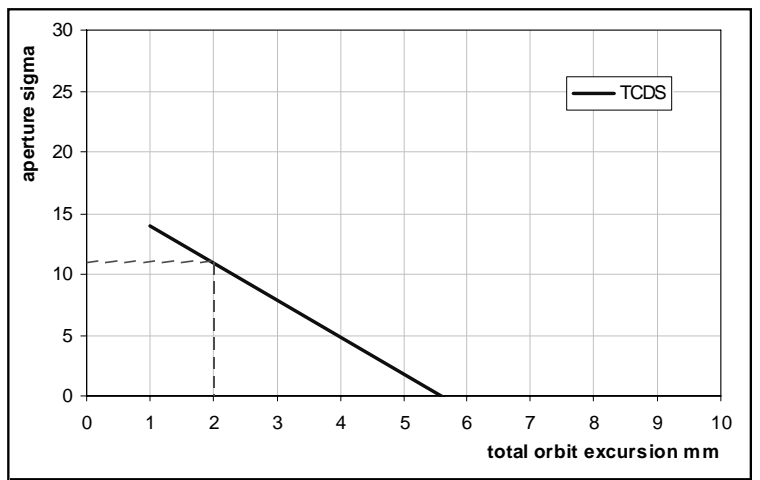

Figure 7. Aperture at $7 \mathrm{TeV}$ for extracted beam as a function of the orbit in the 14/15 MKD case.

\section{APERTURE FOR EXTRACTED BEAM FOR OFF-NORMAL LHC OPERATION}

The dump channel must also accept off-normal LHC operating conditions, manifested as fast orbit excursions and/or an emittance increase. These events are certain to occur during the LHC lifetime, and dump actions are highly likely to be associated with unstable or off-normal beam conditions, so the possible range of parameters must not result in damage to the $\mathrm{LHC}$, including the dumping system itself. Ideally these failures should also not result in any losses in the extraction channel.

\section{TCDS damage limits}

For the various parameters described in table 1, together with the nominal and 14/15 MKD case, the aperture of the dump channel was evaluated and the resulting impacting number of protons on the TCDS calculated and compared to the assumed damage limit. Full details can be found in [2]; the main results are:

- Orbit excursions of up to $\pm 4 \mathrm{~mm}$ should be tolerable without damage, up to nominal intensities,

- Emittance increases of x2 / x4 are safe at $450 \mathrm{GeV}$ / $7 \mathrm{TeV}$ respectively, but may produce $\mathrm{Q} 4$ quenches at low energy for large orbit excursions,

- The 14/15 MKD case will produce losses on the TCDS and a quench of the Q4 magnet.

\section{FURTHER SYSTEM IMPROVEMENTS}

The scope for low-cost improvement is limited since the extraction channel design is essentially finished, tunnelling is completed and hardware construction well advanced. However, the possibility of an increase in the aperture of the MSDC magnets via a new vacuum chamber design is still being pursued.

\section{CONSEQUENCES}

The aperture of the dump channel is limited, especially for off-normal operating conditions of the dump system itself or of the LHC machine. The limits have been quantified at different energies and under various realistic failure scenarios. The following points should be retained:

1. Orbit control (feedback) will be needed to achieve sufficient aperture for the circulating beam of $n 1=6.5$,

2. Operation during commissioning and 'early years' is not expected to be limited by the dump aperture,

3. For normal operation, with realistic failure cases, orbit excursions of up to $\pm 4 \mathrm{~mm}$ should be tolerable without damage, up to nominal intensities,

4. Emittance increases of $\mathrm{x} 2 / \mathrm{x} 4$ are safe at $450 \mathrm{GeV} /$ $7 \mathrm{TeV}$ respectively, but may produce $\mathrm{Q} 4$ quenches at low energy for large orbit excursions,

5. The 14/15 MKD case will produce losses on the TCDS and Q4 quench.

6. Reliable interlocking at about $\pm 4 \mathrm{~mm}$ of the local beam position in point 6 is absolutely necessary.

\section{ACKNOWLEDGEMENTS}

The work described in this paper has been carried out in close and productive collaboration with many colleagues involved in the LHC project. Particular thanks are due to E.Weisse, V.Mertens, J.M.Jimenez, M.Sans, R.Schmidt, J.B.Jeanneret, E. Vossenberg, J.Wenninger and N.Mokhov.

\section{REFERENCES}

[1] G.R. Stevenson, CERN/TIS-RP/IR/93-10.

[2] B.Goddard, Proc. XII ${ }^{\text {th }}$ Chamonix Workshop on LHC Performance, 2003.

[3] J.H. Dieperink et al., LHC Project Report 113.

[4] R.Assmann et al., LHC Project Note 293.

[5] A. Drozhdin, Fermilab Project Note FN-0724, 2002.

[6] J.B.Jeanneret, R.Ostojic, LHC Project Note 111.

[7] J.Uythoven, E.Vossenberg, private communication. 